EPJ manuscript No.

(will be inserted by the editor)

\title{
Parallel dynamics of the asymmetric extremely diluted Ashkin-Teller neural network
}

\author{
D. Bollé and G. Jongen \\ Instituut voor Theoretische Fysica, K.U. Leuven, B-3001 Leuven, Belgium \\ e-mail: desire.bolle@fys.kuleuven.ac.be
}

\begin{abstract}
The parallel dynamics of the asymmetric extremely diluted Ashkin-Teller neural network is studied using signal-to-noise analysis techniques. Evolution equations for the order parameters are derived, both at zero and finite temperature. The retrieval properties of the network are discussed in terms of the four-spin coupling strength and the temperature. It is shown that the presence of a four-spin coupling enhances the retrieval quality.
\end{abstract}

PACS. 64.60.Cn Order-disorder transformations; statistical mechanics of model systems - 75.10.Hk Classical spin models - 87.10.+e General, theoretical, and mathematical biophysics - 02.50.-r Probability theory, stochastic processes and statistics

\section{Introduction}

Recently, the equilibrium properties of the Ashkin-Teller neural network (ATNN) have been studied in [1,2]. The neurons of the ATNN are described by two Ising spins of different types. This allows the network to store and to retrieve pairs of patterns. Therefore, more complicated information can be stored in the ATNN than in the Hopfield model [3, 3 ], e.g., the fore- and background of a picture. Every spin is connected to spins of the same type. In addition, the neurons are connected to each other. The connections linking the neurons are four-spin couplings, since they connect two pairs of spins, one pair per neuron. This allows the network to retrieve both patterns of a pair simultaneously. One can think of the model as a combination of two Hopfield models, each retrieving one of the patterns. The four-spin coupling is then a connection between both models. The underlying idea is that the simultaneous retrieval of a pair of patterns is easier than the independent retrieval of the patterns in the pair.

There are various reasons for studying this model. The Ashkin-Teller spin glass is related to disordered systems where the disorder evolves on a time scale that can be tuned $[5$. The introduction of a neuron containing different types of spins is also neurobiologicaly motivated by the fact that areas in the brain exist which react to two different kinds of dependent stimuli in such a way that the response to particular combinations of these stimuli is stronger than the response to others [ib]. Finally, in neuropsychological studies on amnesia, it has become appreciated that memory is composed of multiple separate systems which can store different types of informations, e.g., information based on skills and informations based on specific facts or data [7].
In [1,2], the thermodynamic and retrieval properties of the ATNN have been studied using replica-symmetric mean-field theory. In the present paper, we analyse the parallel dynamics of the asymmetric extremely diluted version of the model. Both the way how the system evolves to its equilibrium configuration and the properties of the equilibrium configuration itself are subjects of interest. It is known [8, 9] that the dynamics in symmetric architectures, even in the diluted case, is complicated in a nontrivial way because of correlations between the neuron states. These correlations are caused by feedback loops and common ancestors. In contrast to the Hopfield model, where the dynamics has been solved taking into account all the correlations [10, 11], the presence of two types of spins makes the analysis of the correlations in the ATNN very complicated. The underlying reason is the existence of two sources of correlations. First, feedback loops appear due to the two-spin interaction, as in the Hopfield model. Second, the four-spin coupling causes correlations between spins of different type. Therefore, in order to arrive at a first insight in the dynamics of the model, we limit ourselves to its asymmetric extremely diluted version where all correlations between the neuron states are eliminated [12, 13].

Using standard signal-to-noise analysis techniques (see, e.g, refs. [9, 12, 14]), we find that the local field of the asymmetrically diluted ATNN contains only a normally distributed part, besides the signal. As observed already for the asymmetric diluted Hopfield model [12, the structure of the local field does not change in time. This allows us to write down immediately the complete time evolution of the main overlaps.

The rest of the paper is organised as follows. In the second section, we define the model as an extension of 
D. Bollé, G. Jongen: Parallel dynamics of the asymmetric extremely diluted Ashkin-Teller neural network

the Hopfield model. We introduce parallel dynamics at arbitrary temperature, and define the main overlaps (one for each type of spins) as macroscopic measures for the retrieval quality. In Section 3 , we use signal-to-noise analysis techniques in order to write down the evolution equations at arbitrary time. From the evolution equations, the fixed-point equations are obtained. These equations lead to the dynamical capacity-temperature diagram presented in Section 1. Finally, we give some concluding remarks in Section 5 .

\section{The model}

The ATNN is defined as a neural network consisting of $N$ neurons. Each of the neuron states is described by two spins with value $\sigma_{i}$ and $s_{i}(i=1, \ldots, N)$, both taken from the discrete set $\{-1,+1\}$. For each type, the spins $i$ and $j$ are coupled by a two-spin interaction $J_{i j}^{(1)}$ and $J_{i j}^{(2)}$ respectively, while the neurons $i$ and $j$ are coupled by a four-spin interaction $J_{i j}^{(3)}$. We assume no diagonal terms viz. $J_{i i}^{(y)}=0, y=1,2,3$.

A configuration of an ATNN consists out of a $\boldsymbol{\sigma}$ - and s-part viz.

$$
\left(\boldsymbol{\sigma}(t)=\left\{\sigma_{j}(t)\right\}, \mathbf{s}(t)=\left\{s_{j}(t)\right\}\right) ; j=1, \ldots, N .
$$

Given such a configuration, we define three types of local fields: two Hopfield-like local fields which measure the incoming signal to the spins $\sigma_{i}$ and $s_{i}$, caused by the spins of the same type

$$
\begin{aligned}
& h_{N, i}^{(1)}(\boldsymbol{\sigma}(t))=\sum_{j=1}^{N} J_{i j}^{(1)} \sigma_{j}(t) \\
& h_{N, i}^{(2)}(\mathbf{s}(t))=\sum_{j=1}^{N} J_{i j}^{(2)} s_{j}(t),
\end{aligned}
$$

and, in addition, a local field which measures the incoming signal to neuron $i$, caused by both spins of the other neurons

$$
h_{N, i}^{(3)}(\boldsymbol{\sigma}(t), \mathbf{s}(t))=\sum_{j=1}^{N} J_{i j}^{(3)} \sigma_{j}(t) s_{j}(t)
$$

In the sequel, we write the shorthand notation $h_{N, i}^{(x)}(t) \equiv$ $h_{N, i}^{(x)}\left(\mathbf{S}_{x}(t)\right), x=1,2$ with $\mathbf{S}_{1}(t)=\boldsymbol{\sigma}(t), \mathbf{S}_{2}(t)=\mathbf{s}(t)$, and $h_{N, i}^{(3)}(t) \equiv h_{N, i}^{(3)}(\boldsymbol{\sigma}(t), \mathbf{s}(t))$. The configuration $(\boldsymbol{\sigma}(0), \mathbf{s}(0))$ is chosen as input. At temperature $T=1 / \beta$, all neurons are updated in parallel according to the transition probability

$$
\begin{aligned}
& \operatorname{Pr}\left(\sigma_{i}(t+1)=\sigma \mid \boldsymbol{\sigma}(t), \mathbf{s}(t)\right) \\
& \quad=\frac{1}{2}\left[1+\tanh \beta \sigma\left(h_{N, i}^{(1)}(t)+s_{i}(t) h_{N, i}^{(3)}(t)\right)\right] \\
& \operatorname{Pr}\left(s_{i}(t+1)=s \mid \boldsymbol{\sigma}(t), \mathbf{s}(t)\right) \\
& \quad=\frac{1}{2}\left[1+\tanh \beta s\left(h_{N, i}^{(2)}(t)+\sigma_{i}(t) h_{N, i}^{(3)}(t)\right)\right] .
\end{aligned}
$$

We assume hereby that both types of spins exhibit the same degree of stochasticity. At zero temperature, this dynamics becomes deterministic and is given by

$$
S_{x, i}(t+1)=\operatorname{sign}\left(h_{N, i}^{(x)}(t)+S_{\tilde{x}, i}(t) h_{N, i}^{(3)}(t)\right)
$$

with $x, \tilde{x}=1,2$ and $x \neq \tilde{x}$. The $\boldsymbol{\sigma}$-spins receive at each time input from the s-spins and vice versa due to the term containing $h_{N, i}^{(3)}(t)$.

The aim of the network is to store simultaneously $p_{1}$ patterns $\left\{\boldsymbol{\xi}^{\mu}\right\}, \mu=1, \ldots, p_{1}$ in the $\boldsymbol{\sigma}$-part of the network and $p_{2}$ patterns $\left\{\boldsymbol{\eta}^{\mu}\right\}, \mu=1, \ldots, p_{2}$ in the s-part. All components of the patterns $\xi_{i}^{\mu}$ and $\eta_{i}^{\mu}$ are i.i.d.r.v. taken from $\{-1,+1\}$ with zero mean $\left\langle\xi_{i}^{\mu}\right\rangle=0=\left\langle\eta_{i}^{\mu}\right\rangle$ and independent type by type $\left\langle\xi_{i}^{\mu} \eta_{j}^{\nu}\right\rangle=0(i, j=1, \ldots, N)$. In order to store these embedded patterns, the two-spin couplings are chosen according to the Hebb rule

$$
J_{i j}^{(1)}=\frac{J_{1}}{N} \sum_{\mu=1}^{p_{1}} \xi_{i}^{\mu} \xi_{j}^{\mu} \quad J_{i j}^{(2)}=\frac{J_{2}}{N} \sum_{\mu=1}^{p_{2}} \eta_{i}^{\mu} \eta_{j}^{\mu} .
$$

The four-spin interaction is, also in analogy to the Hebbrule, defined as $[1]$

$$
J_{i j}^{(3)}=\frac{J_{3}}{N} \sum_{\mu=1}^{p_{3}} \gamma_{i}^{\mu} \gamma_{j}^{\mu}
$$

Under the assumption of independent embedded patterns $\boldsymbol{\xi}^{\mu}$ and $\boldsymbol{\eta}^{\mu}$, we consider the following form

$$
\gamma_{i}^{\mu}=\xi_{i}^{\mu} \eta_{i}^{\mu}
$$

The patterns $\left\{\gamma^{\mu}\right\}, \mu=1, \ldots, p_{3}$ are then a set of i.i.d.r.v. taken from $\{-1,+1\}$ with zero mean. In the literature, this choice of patterns is called the linked case [1].

The variables $J_{y}, y=1,2,3$ are constants scaling the relative importance of all types of couplings. We choose in the sequel $J_{1}=J_{2}$ since we want both types of spins interchangeable for simplicity. The relative scale of the temperature and coupling strengths is fixed by choosing $J_{1}=1$. Finally, we define $J=J_{3} / J_{1}$ such that this quantity measures the relative strength of the four-spin couplings with respect to the two-spin couplings. In the limit $J \rightarrow 0$ the ATNN becomes, at least in structure, the equivalent of two independent Hopfield models since $h_{N, i}^{(3)}(t)=0$ at all times. We use the temperature $T$ and the relative coupling strength $J$ as independent variables.

In the sequel, we take the interactions asymmetric extremely diluted [12, 13 ]

$$
\tilde{J}_{i j}^{(y)}=c_{i j}^{(y)} N J_{i j}^{(y)} / c_{y} \quad y=1,2,3
$$

with $c_{y}>0$ and $\operatorname{Pr}\left\{c_{i j}^{(y)}=a\right\}=\left(1-c_{y} / N\right) \delta_{a, 0}+\left(c_{y} / N\right) \delta_{a, 1}$. The variables $\left\{c_{i j}^{(y)}\right\}$ are independent for each pair $(i, j)$ representing both the asymmetry and the dilution. The diagonal terms are excluded $c_{i i}^{(y)}=0$. The structure of the architecture of the network then becomes a directed tree 
with an average number of incoming and outgoing connections (type by type) both equal to $c_{y}$. It is assumed that $c_{y} \ll N$. The system is first diluted by taking the limit $N \rightarrow \infty$. Afterwards, the number of incoming signals per site is made extensive by taking the limit $c_{y} \rightarrow \infty$. The probability to have feedback in the system is now zero and the correlations are treelike.

In principle, all couplings can be diluted independently (viz. $c_{1} \neq c_{2} \neq c_{3}$ ). For convenience, however, we dilute them in the same way,

$$
c_{i j}^{(1)}=c_{i j}^{(2)}=c_{i j}^{(3)} \equiv c_{i j} \quad c_{1}=c_{2}=c_{3} \equiv c .
$$

This means that both spins of a neuron get information from the same neurons and that the dynamics of both types of spins can be treated analogously. Since the number of embedded patterns is of the same order as the number of connections a spin has with spins of the same type, we have $p_{1}=p_{2}=p_{3}=\alpha c \equiv p$. In what follows, we write, for simplicity, $J_{i j}^{(y)}$ instead of $\tilde{J}_{i j}^{(y)}$.

At this point, we note that the capacity of the ATNN is defined as the ratio of the number of patterns stored in the network and the number of couplings to a neuron. In this model where we want to store $2 p$ patterns $\left\{\boldsymbol{\xi}^{\mu}, \gamma^{\mu}\right\}$, all neurons have in average $3 c$ links to the other neurons: $2 c$ two-spin couplings and $c$ four-spin couplings. Therefore, the capacity of the ATNN equals

$$
\alpha_{\mathrm{ATNN}} \equiv \frac{2 p}{3 c}=\frac{2}{3} \alpha
$$

The retrieval quality of the model is measured by the Hamming distance between the microscopic state of the network and the stored patterns

$$
\begin{aligned}
& D_{H}^{\mu}(t) \equiv\left[\sum_{x}\left(d_{x}\left(\boldsymbol{\psi}_{x}^{\mu}, \mathbf{S}_{x}(t)\right)\right)^{2}\right]^{\frac{1}{2}} \\
& d_{x}\left(\boldsymbol{\psi}_{x}^{\mu}, \mathbf{S}_{x}(t)\right) \equiv \frac{1}{N} \sum_{i=1}^{N}\left[\psi_{x, i}^{\mu}-S_{x, i}(t)\right]^{2}
\end{aligned}
$$

where $\mu=1, \ldots, p, x=1,2, \boldsymbol{\psi}_{1}^{\mu}=\boldsymbol{\xi}^{\mu}$ and $\boldsymbol{\psi}_{2}^{\mu}=\boldsymbol{\eta}^{\mu}$. This naturally introduces the main overlaps

$$
m_{x, N}^{\mu}(t)=\frac{1}{N} \sum_{i=1}^{N} \psi_{x, i}^{\mu} S_{x, i}(t) \quad \mu=1, \ldots, p .
$$

In the diluted model the sum in (14) has to be taken over the tree-like structure, viz. $\frac{1}{N} \sum_{i=1}^{N} \rightarrow \frac{1}{c} \sum_{i=1}^{N} c_{i j}$. The expression for the main overlap (14) then reads

$$
m_{x, c, N}^{\mu}(t)=\frac{1}{c} \sum_{i=1}^{N} c_{i j} \psi_{x, i}^{\mu} S_{x, i}(t) \quad \mu=1, \ldots, p
$$

We remark that both expressions (14) and (15) become equal in the thermodynamic limit $c, N \rightarrow \infty$.

\section{Dynamics}

In this section we construct a set of recursion equations for the main overlap order parameters. We use hereby signal-to-noise techniques (see, e.g., refs. 13, 14). Finally, we write down the fixed-point equations.

Suppose an initial spin configuration $(\boldsymbol{\sigma}(0), \mathbf{s}(0))$. The configurations $\mathbf{S}_{x}(0)=\left\{S_{x, i}(0)\right\}, i=1, \ldots, N$ are collections of i.i.d.r.v. with mean $\left\langle S_{x, i}(0)\right\rangle=0$ and variance $\left\langle\left(S_{x, i}(0)\right)^{2}\right\rangle=1$. Spins of different types are uncorrelated $\left\langle\sigma_{i}(0) s_{j}(0)\right\rangle=0(i, j=1, \ldots, N)$. Both types are correlated with only one of the stored patterns, e.g., the first one

$$
\left\langle\psi_{x, i}^{\mu} S_{x, j}(0)\right\rangle=\delta_{i, j} \delta_{\mu, 1} m_{x, 0}^{1} .
$$

The site by site independence of spins and patterns implies by the law of large numbers (LLN) that we get for the main overlaps

$$
m_{x}^{1}(0) \equiv \lim _{c, N \rightarrow \infty} m_{x, c, N}^{1}(0)=\left\langle\psi_{x, i}^{1} S_{x, i}(0)\right\rangle=m_{x, 0}^{1}
$$

We now want to study how the main overlaps evolve under the parallel dynamics specified before. For a general time step and at $T=0$, we find from (15) and the LLN in the limit $c, N \rightarrow \infty$

$$
\begin{aligned}
& m_{x}^{1}(t+1)=\left\langle\left\langle\psi_{x, i}^{1} \operatorname{sign}\left(h_{i}^{(x)}(t)+\psi_{\tilde{x}, i}(t) h_{i}^{(3)}(t)\right)\right\rangle\right\rangle \\
& m_{3}^{1}(t+1)=\left\langle\left\langle\xi_{i}^{1} \eta_{i}^{1} \operatorname{sign}\left(h_{i}^{(1)}(t)+s_{i}(t) h_{i}^{(3)}(t)\right)\right.\right. \\
&\left.\left.\quad \times \operatorname{sign}\left(h_{i}^{(2)}(t)+\sigma_{i}(t) h_{i}^{(3)}(t)\right)\right\rangle\right\rangle(18
\end{aligned}
$$

where $x, \tilde{x}=1,2 ; x \neq \tilde{x}$. The average $\langle\langle\cdot\rangle\rangle$ denotes the average both over the distribution of the embedded patterns $\left\{\xi_{i}^{\mu}\right\}$ and $\left\{\eta_{i}^{\mu}\right\}$ and the initial configuration $\left\{\sigma_{i}(0), s_{i}(0)\right\}$. The average over the latter is hidden in an average over the local fields through the updating rule (4).

The equations (18) show that the knowledge of the distribution of the focal field at successive time steps is sufficient in order to find the evolution equations for the order parameters. We start with calculating the distribution of the local field of the $\boldsymbol{\sigma}$-spins at $t=0$. Using the definitions (7) and applying the signal-to-noise analysis, we have

$$
h_{N, i}^{(1)}(0)=\xi_{i}^{1} \frac{1}{c} \sum_{j=1}^{N} c_{i j} \xi_{j}^{1} \sigma_{j}(0)+\frac{1}{c} \sum_{\mu=2}^{p} \xi_{i}^{\mu} \sum_{j=1}^{N} c_{i j} \xi_{j}^{\mu} \sigma_{j}(0) .
$$

The signal term, i.e., the first term on the r.h.s. of (19), is nothing but the main overlap (15) multiplied by $\xi_{i}^{1}$. In the noise part, i.e., the second term on the r.h.s., all terms are uncorrelated by construction such that we can apply the central limit theorem (CLT) to find

$$
\lim _{c, N \rightarrow \infty} \frac{1}{\sqrt{p}} \sum_{\mu=2}^{p} \xi_{i}^{\mu} \frac{1}{\sqrt{c}} \sum_{j=1}^{N} c_{i j} \xi_{j}^{\mu} \sigma_{j}(0) \sim \mathcal{N}(0,1)
$$


where $\mathcal{N}(0,1)$ represents a Gaussian random variable with mean 0 and variance 1 . Therefore, in the limit $c, N \rightarrow \infty$, the local field at $t=0$ is the sum of two independent random variables

$$
h_{i}^{(1)}(0) \equiv \lim _{c, N \rightarrow \infty} h_{N, i}^{(1)}(0)=\xi_{i}^{1} m_{1}^{1}(0)+\sqrt{\alpha} z_{1}(0)
$$

with $z_{1}(0) \sim \mathcal{N}(0,1)$. In an analogous way, we find for the local field of the s-spins

$$
h_{i}^{(2)}(0) \equiv \lim _{c, N \rightarrow \infty} h_{N, i}^{(2)}(0)=\eta_{i}^{1} m_{2}^{1}(0)+\sqrt{\alpha} z_{2}(0)
$$

with $z_{2}(0) \sim \mathcal{N}(0,1)$.

As in the local fields of the spins (2), we separate in the local field (3) the terms containing the first pattern $\gamma^{1}$ from the rest

$$
\begin{aligned}
h_{N, i}^{(3)}(0)=\gamma_{i}^{1} & \frac{1}{c} \sum_{j=1}^{N} c_{i j} \gamma_{j}^{1} \sigma_{j}(0) s_{j}(0) \\
& +\frac{1}{c} \sum_{\mu=2}^{p} \gamma_{i}^{\mu} \sum_{j=1}^{N} c_{i j} \gamma_{j}^{\mu} \sigma_{j}(0) s_{j}(0) .
\end{aligned}
$$

In analogy with before, we call the first term the signal and the last term the noise. Applying the LLN to the signal term, we get

$$
\lim _{c, N \rightarrow \infty} \frac{1}{c} \sum_{j=1}^{N} c_{i j} \gamma_{j}^{1} \sigma_{j}(0) s_{j}(0)=\left\langle\gamma_{j}^{1} \sigma_{j}(0) s_{j}(0)\right\rangle .
$$

Since this term resembles strongly the main overlaps (15), we call it also an overlap and denote it by $m_{3}^{1}(0)$. In general, this overlap is defined by

$$
m_{3, c, N}^{\mu}(t)=\frac{1}{c} \sum_{i=1}^{N} c_{i j} \gamma_{i}^{\mu} \sigma_{i}(t) s_{i}(t) \quad \mu=1, \ldots, p .
$$

In the sequel, we will treat this parameter at the same level as the other overlaps (15). For the linked choice of patterns $\gamma_{i}^{\mu} \equiv \xi_{i}^{\mu} \eta_{i}^{\mu}$, it follows from (16) that $\left\langle\gamma_{i}^{\mu} \sigma_{j}(0) s_{k}(0)\right\rangle=$ $\delta_{i j} \delta_{i k} \delta_{\mu 1} m_{1,0}^{1} m_{2,0}^{1}$ since the initial spin configurations $\sigma_{j}(0)$ and $s_{j}(0)$ are independent. Therefore, we have in the thermodynamic limit and at $t=0$

$$
m_{3}^{1}(0) \equiv \lim _{c, N \rightarrow \infty} m_{3, c, N}^{1}(0)=m_{1}^{1}(0) m_{2}^{1}(0) .
$$

Following the same line of arguments as before, the noise term converges again to a Gaussian random variable such that

$$
h_{i}^{(3)}(0) \equiv \lim _{c, N \rightarrow \infty} h_{N, i}^{(3)}(0)=J \xi_{i}^{1} \eta_{i}^{1} m_{3}^{1}(0)+J \sqrt{\alpha} z_{3}(0)
$$

with $z_{3}(0) \sim \mathcal{N}(0,1)$. This finishes the calculation of the local fields at time $t=0$.

At a general time $t$, the local fields still consist out of a signal term, proportional to the main overlap, and a Gaussian distributed noise part. This is due to the extreme dilution which eliminates all common ancestors in the dynamics. Therefore, all variables $\left\{X_{j}^{\mu} \equiv \psi_{x, i}^{\mu} c_{i j} \psi_{x, j}^{\mu} S_{x, j}(t) \mid j=\right.$
$1, \ldots, N ; \mu=1, \ldots, p\}$ are a set of i.i.d.r.v. and we can apply the CLT in the same way as in eq. (19). So, we find for the distribution of the local fields a set of equations with the same structure as the eqs. (21), (22) and (27), viz.

$$
\begin{aligned}
& h_{i}^{(x)}(t)=\psi_{x, i}^{1} m_{x}^{1}(t)+\sqrt{\alpha} z_{x}(t) ; z_{x}(t) \sim \mathcal{N}(0,1) \\
& h_{i}^{(3)}(t)=J \xi_{i}^{1} \eta_{i}^{1} m_{3}^{1}(t)+J \sqrt{\alpha} z_{3}(t) ; z_{3}(t) \sim \mathcal{N}(0,1) .
\end{aligned}
$$

The three Gaussian variables $z_{y}(t)$ are uncorrelated.

Using the distributions of the local fields (28) and remarking that the joint probability of $s_{i}(t), \sigma_{i}(t), \xi_{i}^{1}$ and $\eta_{i}^{1}$ is obtained from the overlaps $m_{y}^{1}(t), y=1,2,3$, the equations (18) and (28) lead immediately to the evolution equations for the order parameters at zero temperature

$$
\begin{array}{r}
m_{x}^{1}(t+1)=\sum_{\sigma= \pm 1} \frac{1}{2}\left(1+\sigma m_{\tilde{x}}^{1}(t)\right) \operatorname{Erf}\left(\frac{m_{x}^{1}(t)+\sigma J m_{3}^{1}(t)}{\sqrt{2 \alpha\left(1+J^{2}\right)}}\right) \\
m_{3}^{1}(t+1)=\sum_{\sigma, \tau= \pm 1}\left(\sigma \tau+\sigma m_{1}^{1}(t)+\tau m_{2}^{1}(t)+m_{3}^{1}(t)\right) \\
\quad \int \mathcal{D} z \operatorname{Erf}\left(\frac{\sigma m_{1}^{1}(t)+J m_{3}^{1}(t)+J \sqrt{\alpha} z}{\sqrt{2 \alpha}}\right) \\
\quad \times \operatorname{Erf}\left(\frac{\tau m_{2}^{1}(t)+J m_{3}^{1}(t)+J \sqrt{\alpha} z}{\sqrt{2 \alpha}}\right)
\end{array}
$$

where $x, \tilde{x}=1,2, x \neq \tilde{x}$.

After some time $t$ the dynamics reaches the point where the spins macroscopically equilibrate. This means that the main overlap becomes stationary, viz. $m_{y}^{1}(t+1)=m_{y}^{1}(t)$. Since the expressions for the local fields do not change their structure, the corresponding fixed-point equations are easily obtained from eq. (29) by replacing the time dependent quantities by their equilibrium value $m_{y}^{1} \equiv$ $\lim _{t \rightarrow \infty} m_{y}^{1}(t)$. In the limit $J \rightarrow 0$, the equations (29) are consistent with the evolution equations of [12].

At non-zero temperature $T=1 / \beta$ the main overlaps at time $t$ read

$$
\begin{aligned}
& m_{x}^{1}(t+1)=\left\langle\left\langle\psi_{x, i}^{1}\left\langle S_{x, i}(t+1)\right\rangle_{\beta}\right\rangle\right\rangle \\
& m_{3}^{1}(t+1)=\left\langle\left\langle\xi_{i}^{1} \eta_{i}^{1}\left\langle\sigma_{i}(t+1)\right\rangle_{\beta}\left\langle s_{i}(t+1)\right\rangle_{\beta}\right\rangle\right\rangle .
\end{aligned}
$$

The thermal averages are defined by the updating rule (4) and can be written as

$$
\left\langle S_{x, i}(t+1)\right\rangle_{\beta}=\tanh \left[\beta\left(h_{i}^{(x)}(t)+S_{\tilde{x}, i}(t) h_{i}^{(3)}(t)\right)\right] .
$$

The stochasticity in the dynamics does not modify the local spin correlations when compared with the deterministic dynamics. Therefore, the local fields are still distributed according to (28) and we get for the order parameters

$$
m_{x}^{1}(t+1)=\sum_{\sigma= \pm 1} \frac{1}{2}\left(1+\sigma m_{\tilde{x}}^{1}(t)\right)
$$




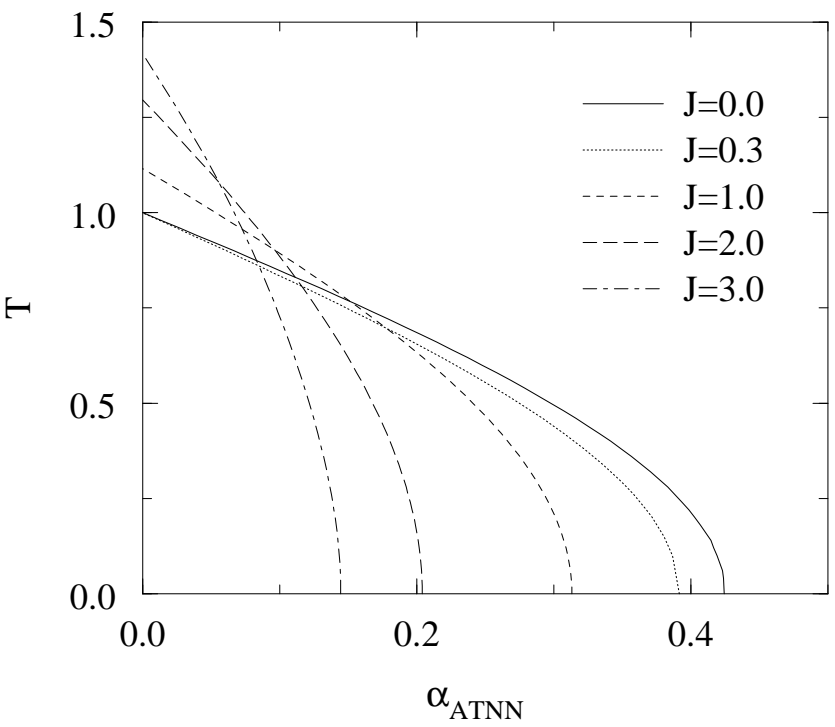

Fig. 1. Capacity-temperature diagram for the ATNN for $\mathrm{J}=0.0$ (full line), $J=0.3$ (dotted line), $J=1.0$ (dashed line), $J=2.0$ (long dashed line) and $J=3.0$ (dot-dashed line).

$$
\begin{aligned}
& \times \int \mathcal{D} y \tanh \beta\left(m_{x}^{1}(t)+\sigma J m_{3}^{1}(t)+\sqrt{\alpha\left(1+J^{2}\right)} y\right) \\
& m_{3}^{1}(t+1)=\sum_{\sigma, \tau= \pm 1} \frac{1}{4}\left(\sigma \tau+\sigma m_{1}^{1}(t)+\tau m_{2}^{1}(t)+m_{3}^{1}(t)\right) \\
& \times \int \mathcal{D} z \int \mathcal{D} x \tanh \beta\left(\sigma m_{1}^{1}(t)+J m_{3}^{1}(t)+\sqrt{\alpha} x+J \sqrt{\alpha} z\right) \\
& \times \int \mathcal{D} y \tanh \beta\left(\tau m_{2}^{1}(t)+J m_{3}^{1}(t)+\sqrt{\alpha} y+J \sqrt{\alpha} z\right) \cdot(32)
\end{aligned}
$$

The fixed-point equations are read off from (32) by using again $m_{y}^{1}=\lim _{t \rightarrow \infty} m_{y}^{1}(t), y=1,2,3$. In the zerotemperature limit $\beta \rightarrow \infty$, the equations above reduce to (29). Moreover, in the limit $J \rightarrow 0$, they are consistent with the ones obtained for the asymmetric extremely diluted Hopfield model in 12 .

\section{Results}

In this section, we discuss the numerical results for the ATNN obtained from the fixed-point equations derived in the previous section. We present the capacity-temperature diagram indicating the regions of retrieval as a function of the capacity $\alpha_{\mathrm{ATNN}}$ and the temperature $T$, and some representative figures illustrating the main features of the model.

Due to the choice $J_{1}=J_{2}=1$ and due to the condition that both types of spins have a finite initial overlap with one condensed pattern, it turns out that the overlaps $m_{1}(t)$ and $m_{2}(t)$ always converge to the same equilibrium values, independent of the size of the initial overlaps. (We forget about the superscript 1 ). Therefore we can restrict ourselves to the case $m_{1}=m_{2}$.
The resulting capacity-temperature diagram is presented in Fig. 1. First, we consider the special case $J=0$. At $T=0$ a non-zero solution for the fixed-point equations exists as long as $\alpha<2 / \pi$, indicating a transition from the retrieval to the non-retrieval regime at $\alpha_{\mathrm{ATNN}}=4 / 3 \pi$. When the temperature increases, the critical capacity decreases to become zero at $T=1$. The resulting transition line in the capacity-temperature diagram is similar to the one of the Hopfield model [12] up to a rescaling of the capacity. This is not surprising since the structure of the equations of the ATNN for $J=0$ is consistent with those of the Hopfield model. The transition is always continuous, and the main overlap decreases when $\alpha_{\text {ATNN }}$ increases. This indicates that the more embedded patterns, the harder the retrieval and the worse the retrieval quality.

When the four-spin coupling is non-zero, things become different. A larger four-spin coupling makes retrieval possible at higher temperature and decreases the critical capacity at low temperature.

For finite loading $\left(\alpha_{\mathrm{ATNN}}=0\right)$, a continuous transition occurs for $J \leq 1 / 3$ at $T=1$. For larger $J$, the transition becomes discontinuous and the critical temperature becomes larger. This indicates that a model with non-zero four-spin couplings can perform better in the presence of noise in the dynamics. This corresponds with the results in [1]. The overlap $m_{1}$ at the critical temperature increases up to $J=2$, meaning that the larger $J$ the better the retrieval quality. From $J=2$ onwards, the increasing noise in the dynamics at the transition line results in a slowly decreasing overlap $m_{1}$. We note that we always observe $m_{3}=\left(m_{1}\right)^{2}$ when $\alpha_{\mathrm{ATNN}}=0$.

At zero temperature $(T=0)$, increasing $J$ implies decreasing the critical capacity. The transition is always first order, except for $J=0$. The main overlap $m_{1}$ at the transition line first increases with $J$, but starts to decrease from $J=2.0$ onwards. At larger $J$, it starts increasing again. The overlap $m_{3}$, however, always increases and becomes larger than $m_{1}$ for $J \geq 4.2$. The critical capacity for $J=1$ is equal to 0.3131 , which is higher than that of the fully connected ATNN $\left(\alpha_{c}=0.1839\right)$ [2]. This is consistent with the results obtained by comparing the asymmetric extremely diluted with the fully connected Hopfield model [4,12].

For non-zero temperature and infinite loading, the transition is partially continuous as long as $J \in[0,1 / 3]$. The larger $J$, the larger the temperature is where a continuous transition occurs. As an example, we have drawn the value of the overlaps at the critical capacity for $J=0.3$ (Fig. 2 $2 a$ ) where the transition is continuous for $T \geq 0.88$. When $J \geq 1 / 3$, the transition is discontinuous for all temperature. In Fig. 2 $b$ and Fig. 2 $c$, we have drawn the overlaps at the critical capacity for $J=1$ and $J=3$. The overlap $m_{1}$ exhibits a maximum at $T=0.32$ and $T=0.86$ respectively while $m_{3}$ is always decreasing.

\section{Concluding remarks}

In this article, we have studied the parallel dynamics of the asymmetric extremely diluted ATNN with linked pat- 
D. Bollé, G. Jongen: Parallel dynamics of the asymmetric extremely diluted Ashkin-Teller neural network
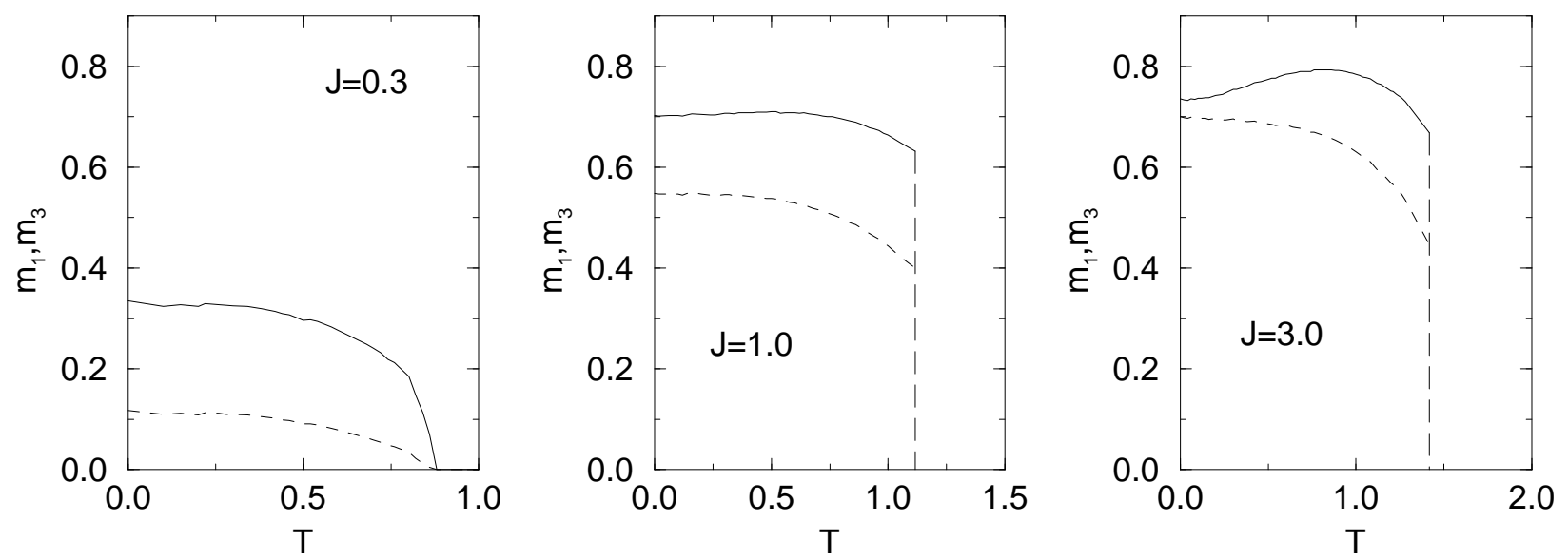

Fig. 2. Overlaps $m_{1}$ (full line) and $m_{3}$ (broken line) at the critical capacity as a function of the temperature, for $a$ ) $\mathrm{J}=0.3, b$ ) $\mathrm{J}=1.0$ and $c$ ) $\mathrm{J}=3.0$.

terns at arbitrary temperature. Because of the absence of correlations between the neurons, we have found that the noise of the local field at all time steps is normally distributed. Hence, the dynamical equations for the order parameters are obtained immediately. Furthermore, the dynamical capacity-temperature diagram is discussed.

In the presence of the four-coupling term, the dynamics can exhibit more noise without disturbing the retrieval process completely. Moreover, the transition from the retrieval to the non-retrieval regime becomes first order. This implies that the Hamming distance becomes smaller, even at the transition line. So in general, we can say that the four-coupling term enhances the retrieval quality of the network.

The authors are indebted to L. Bianchi for some contributions at the initial stages of this work and to P. Kozłowski for constructive discussions. One of us (D.B.) thanks the Fund for Scientific Research - Flanders (Belgium) for financial support.

\section{References}

1. D. Bollé and P. Kozłowski, J. Phys. A: Math. Gen. 31, 6319 (1998).

2. D. Bollé and P. Kozłowski, cond-mat/9906274, (to be published in J. Phys. A: Math. Gen.) (1999).

3. J.J. Hopfield, Proc. Nat. Acad. Sci. USA 79, 2554 (1982).

4. D. Amit, H. Gutfreund and H. Sompolinsky, Ann. Phys. (N. Y.) 173, 30 (1987).

5. N. Caticha, J. Phys. A: Math. Gen. 27, 5501 (1994).

6. H. Komatsu and Y. Ideura, J. Neurophysiol. 70, 677 (1993).

7. L.R. Squire, Brain mechanisms of perception and memory ed. T. Ono et al. (Oxford University Press, 1993), 219; Science 232, 1612 (1986).

8. E. Barkai, I. Kanter and H. Sompolinsky, Phys. Rev. A 41, 590 (1990).

9. D. Bollé, G. Jongen and G.M. Shim, to appear in the Proceedings of the International Conference on Mathematical Physics and Stochastic Analysis (Lisbon, October 1998), ed. S. Albeverio et al. (World Scientific, 1999).
10. D. Bollé, G. Jongen and G.M. Shim, J. Stat. Phys. 91, 125 (1998).

11. D. Bollé, G. Jongen and G.M. Shim, J. Stat. Phys. 96, 861 (1999).

12. B. Derrida, E. Gardner, and A. Zippelius, Europhys. Lett. 4, 167 (1987).

13. D. Bollé, G.M. Shim, B. Vinck, and V.A. Zagrebnov, J. Stat. Phys. 74, 565 (1994).

14. A.E. Patrick and V.A. Zagrebnov, J. Phys. A: Math. Gen. 24, 3413 (1991); J. Stat. Phys. 63, 59 (1991). 